\title{
Qualidade pós-colheita de frutos de tomate cv. Andréa tratados com etileno ${ }^{1}$
}

\section{Caroline Andreuccetti²; Marcos David Ferreira ${ }^{3}$; Celso Luiz Morettii ${ }^{4}$; Sylvio Luis Honório ${ }^{3}$}

${ }^{2}$ Universidade Estadual de Campinas, FEA, C. Postal 6122, 13083-862 Campinas-SP; ${ }^{3}$ Universidade Estadual de campinas, FEAGRI, C. Postal 6011, 13083-875 Campinas-SP; ${ }^{4}$ Embrapa Hortaliças, C. Postal 218, 70359-970 Brasília, DF; E-mail: caroline@ fea.unicamp.br;

\section{RESUMO}

O tomate é uma das principais hortaliças cultivadas no Brasil e tratamentos que propiciem o controle do amadurecimento são extremamente desejáveis. Avaliou-se a qualidade pós-colheita de tomates tipo italiano tratados com etileno. Tomates 'Andréa' foram colhidos em campos de produção comercial no estádio verde-maduro, selecionados para presença de danos externos e classificados quanto ao tamanho e cor e posteriormente foram tratados com 100 $\mu \mathrm{L} \mathrm{L}^{-1}$ de etileno. Os ensaios foram planejados com delineamento inteiramente casualizado em esquema fatorial com quatro tratamentos e quinze repetições $(n=10)$. Após a aplicação, os frutos foram divididos em dois lotes. Um lote permaneceu a $20^{\circ} \pm 1^{\circ} \mathrm{C}$ e outro foi mantido a $12,5^{\circ} \pm 1^{\circ} \mathrm{C}$, ambos sob umidade relativa $90 \pm 5 \%$. Tomates do tratamento controle foram mantidos nas mesmas condições de temperatura e umidade relativa. Os frutos foram avaliados após cada mudança de estádio de amadurecimento para as seguintes variáveis: coloração externa ( $\mathrm{L} * \mathrm{a}^{*} \mathrm{~b} *$ ), perda de massa, sólidos solúveis totais, acidez titulável e teor de ácido ascórbico. A aplicação de etileno não influenciou de maneira significativa as variáveis químicas e físicas estudadas. Frutos armazenados à temperatura de $20^{\circ} \mathrm{C}$ demonstraram maior porcentagem de perda de massa ao longo do amadurecimento, com média de $2,60 \%$. A relação brix/acidez apresentou pouca variação entre os tratamentos avaliados. Frutos do tratamento controle e mantidos à temperatura de $20^{\circ} \mathrm{C}$ apresentaram mudança de coloração mais rápida no início dos experimentos, não sendo observada posteriormente diferença significativa no desenvolvimento da cor dos tratamentos avaliados. O tratamento com etileno não acelerou de forma significativa o amadurecimento de tomates 'Andréa' armazenado sob as duas temperaturas estudadas.

Palavras-chave: Lycopersicon esculentum Mill., fluxo dinâmico, perda de massa, vitamina $\mathrm{C}$.

\section{ABSTRACT}

Postharvest quality of tomato fruits cv. Andréa treated with ethylene

Tomatoes are one of the most important vegetable crops grown in Brazil and treatments that facilitate ripening control are extremely desirable. The present work was carried out aiming to evaluate postharvest quality of roma-type tomatoes treated with ethylene. 'Andréa' tomatoes were harvested in commercial fields at the maturegreen stage, were graded for size and color, and treated with ethylene $\left(100 \mu \mathrm{L} \mathrm{L}^{-1}\right)$ for 48 hours at $20^{\circ} \pm 1^{\circ} \mathrm{C}$ and relative humidity of $90 \pm 5 \%$.

The experiments were carried out using a completely randomized design with four treatments and fifteen replicates $(n=10)$. After ethylene application, tomatoes were stored at two temperatures. One lot remained at $20^{\circ} \pm 1^{\circ} \mathrm{C}$ and another one was kept at $12.5^{\circ} \pm 1^{\circ} \mathrm{C}$ and relative humidity of $90 \pm 5 \%$. Control fruits were kept in the same storage conditions of temperature and relative humidity. For each maturity color change, the following analyses were carried out: color indexes ( $\mathrm{L}^{*}, \mathrm{a}^{*}$ and the $\mathrm{b} *$ and its relations), water loss $(\%)$, soluble solids ( ${ }^{\circ}$ Brix), titratable acidity $\left(\mathrm{g} 100 \mathrm{~g}^{-1}\right)$, and ascorbic acid (mg $\left.100 \mathrm{~g}^{-1}\right)$. Ethylene application promoted color uniformity in treated fruits. However, there were no significant changes in the other postharvest variables evaluated. Fruits stored at $20^{\circ} \mathrm{C}$ showed $2.6 \%$ of mass loss during storage. Brix/titratable ratio showed slight variation throughout the experiment. Control fruits stored at $20^{\circ} \mathrm{C}$ changed external color earlier than other treatments in the beginning of the trial, showing no significant difference after that. Postharvest ethylene application was not an efficient technique to hasten ripening of 'Andréa' tomatoes.

Keywords: Lycopersicon esculentum Mill., flow through system, mass loss, vitamin C.

\section{(Recebido para publicação em 24 de março de 2006; aceito em 26 de fevereiro de 2007)}

$\mathrm{O}$ tomate é amplamente cultivado e utilizado no Brasil, podendo ser consumido ao natural ou na forma processada. Vários estados brasileiros produzem tomate com destaque para Goiás, São Paulo e Minas Gerais, os quais atingiram respectivamente, no ano de 2003, a produção de 1.017, 768 e 693 mil toneladas (MAPA, 2005). Os frutos possuem significativas quantidades de pigmentos carotenóides, tiamina, niacina e vitamina C (Stevens, 1985).
O tomate caracteriza-se por ser um fruto climatérico e seu amadurecimento normalmente se inicia na porção distal do fruto, migrando para as regiões vizinhas pelo processo de difusão livre até que o processo de amadurecimento atinja todo o fruto (Reid, 1994; Alexander $\&$ Grierson, 2002). Esse efeito é claramente percebido pela mudança de coloração no fruto que se inicia no gel locular, ao redor das sementes e depois migra para o pericarpo progressivamente (Hobson \& Grierson, 1993).
O etileno é o único hormônio gasoso presente nas plantas e possui diferentes efeitos nas células vegetais, promovendo alterações metabólicas nos diversos estádios de desenvolvimento dos tecidos vegetais. Alguns dos efeitos conhecidos são crescimento e diferenciação celular, formação de raízes adventícias, germinação de sementes, indução da floração em algumas espécies (por exemplo, o abacaxizeiro), abscisão e senescência de folhas, flores e frutos. $\mathrm{O}$ etileno também está envolvido nos pro-

\footnotetext{
${ }^{1}$ Parte do projeto de mestrado, com apoio financeiro da FAPESP e do sistema PRODETAB/EMBRAPA.
} 
cessos de resposta a estresses de caráter biótico ou abiótico como ataques de patógenos e ferimentos nos tecidos vegetais (Mckeon \& Yang, 1987; Moretti et al., 1998; Saltveit, 1999; Kieber, 2004). O etileno também está diretamente relacionado com o processo de amadurecimento dos frutos. Por isso, comercialmente, pode ser utilizado como meio de acelerar, controlar e uniformizar o amadurecimento de diferentes órgãos de várias espécies vegetais.

Basicamente, o etileno na forma gasosa pode ser aplicado por meio de um sistema estático ou dinâmico. No sistema estático o produto é colocado no interior de um recipiente com volume conhecido por um período determinado de tempo, no qual o gás é injetado. Já o sistema dinâmico consiste num fluxo contínuo de gás (ou gases) que entram em contato com o produto a ser tratado. Em qualquer que seja o sistema adotado, os parâmetros de aplicação de etileno devem ser garantidos de forma a proporcionar amadurecimento adequado e uniforme aos frutos de tomate. Os parâmetros consistem na concentração, tempo de exposição ao gás e condições ambientais, como por exemplo, recomendações de temperatura e umidade relativa. Normalmente a concentração de etileno recomendada para tomates é $100 \mathrm{~mL} \mathrm{~L}^{-1}$ com tempo de aplicação equivalente a 24-48 horas (Gull, 1981; Hobson \& Grierson, 1993; Reid, 2002; Suslow \& Cantwell 2003; Sargent \& Moretti, 2004).

Como o mercado de tomates em todo o mundo é muito dinâmico e anualmente surgem novos híbridos e cultivares, a avaliação do comportamento de novos materiais ao etileno exógeno é extremamente desejável. Existe uma lacuna na literatura consultada sobre o comportamento de tomates tipo italiano 'Andréa' em relação ao etileno exógeno. A partir desse contexto, o presente trabalho teve por objetivo avaliar a qualidade póscolheita de frutos de tomate cv. Andréa tratados com etileno.

\section{MATERIAL E MÉTODOS}

Frutos de tomate (Lycopesicon esculentum Mill) da cultivar Andréa foram colhidos no estádio verde-maduro em campos de produção comercial no município de Apiaí (SP), durante o mês de maio de 2004. Os frutos colhidos foram transportados em caixas plásticas durante o período noturno para uma unidade de beneficiamento situada no município de Cabreúva (SP).

Os frutos foram selecionados na unidade de beneficiamento passando pelas seguintes etapas: recebimento; lavagem, secagem e escovação; classificação e embalagem. A classificação foi realizada eletronicamente por diâmetro e cor, de acordo com as normas do MAPA (2000). Após o beneficiamento, os frutos foram transportados para o laboratório da Faculdade de Engenharia Agrícola (Unicamp), em Campinas, SP, no qual passaram por nova seleção, sendo descartados os tomates com início de coloração pintando e frutos brocados.

A aplicação do gás etileno foi realizada em fluxo dinâmico, utilizando-se uma mistura gasosa composta por etil $5 \%\left(50.000 \mu \mathrm{L} \mathrm{L}^{-1}\right)$ e ar comprimido, à temperatura de $20^{\circ} \pm 1^{\circ} \mathrm{C}$, sob umidade relativa de $90 \pm 5 \%$, por 48 horas. A concentração da mistura contendo etileno foi mantida a $100 \mu \mathrm{L} \mathrm{L}^{-1}$. Após o tratamento com etileno os frutos foram divididos em dois lotes. Os frutos tratados ou não com etileno foram armazenados sob duas temperaturas $\left(20^{\circ} \pm 1^{\circ} \mathrm{C}\right.$ e a $12,5^{\circ} \pm 1^{\circ} \mathrm{C}$ ) e umidade relativa de $90 \pm 5 \%$.

A periodicidade das análises físicas e químicas deu-se de acordo com a percepção visual da mudança na coloração dos frutos armazenados, considerandose como mudança de coloração quando $60 \%$ dos frutos ou mais de um mesmo tratamento atingissem o estádio de amadurecimento subseqüente, de acordo com a classificação adotada pelo Ministério da Agricultura, Pecuária e Abastecimento (MAPA, 2000). Foram considerados cinco estádios de amadurecimento: verde-maduro, pintando, rosado, vermelho e vermelho-maduro.

A perda de massa foi calculada em relação ao peso inicial do fruto com o auxílio de uma balança Marte, modelo AL 500, com 0,01 g de precisão e $500 \mathrm{~g}$ de carga máxima. Para a análise de cor foi utilizado um espectrocolorímetro Hunter Lab., com miniscan XE Plus, parâmetros D65/10 ${ }^{\circ}$. Foram feitas me- dições num mesmo fruto, na região equatorial em três faces opostas. Os resultados foram expressos pela escala $\mathrm{L}^{*}$, $a^{*}$ e b*. Na análise de acidez titulável foram adotados os procedimentos descritos por Carvalho et al. (1990), com resultados expressos em gramas de ácido cítrico por $100 \mathrm{~g}$ de suco de tomate

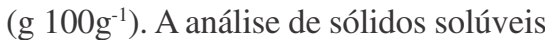
foi realizada com um refratômetro Abbe Refractometer, tipo WYA. Essa análise realizou-se de acordo com procedimentos descritos por Carvalho et al. (1990). Essa determinação foi dada em graus Brix ( ${ }^{\circ}$ Brix). Para a determinação do teor de ácido ascórbico foi utilizado o método padrão da AOAC (1984), modificado por Benassi \& Antunes (1988), por meio de titulação com 2,6 dichlorophenol-indophenol. Os resultados foram expressos em $\mathrm{mg}$ de ácido ascórbico. $100 \mathrm{~g}^{-1}$ de matéria fresca.

Os ensaios foram planejados com delineamento inteiramente casualizado em esquema fatorial ( 2 X 2 ) com quinze repetições $(n=10)$. Os fatores considerados foram a aplicação do gás etileno (dois tratamentos, um com etileno outro sem etileno) e duas temperatura de armazenamento $\left(12,5^{\circ} \mathrm{C}\right.$ e $\left.20^{\circ} \mathrm{C}\right)$. Os dados foram submetidos à análise de variância e as médias foram comparadas pelo teste de diferença mínima significativa em teste de comparações múltiplas, em que as diferenças entre dois tratamentos maior que a soma de dois desvios-padrões foram consideradas significativas ao nível de 5\% de probabilidade (Shamaila et al., 1992).

\section{RESULTADOS E DISCUSSÃO}

Verificou-se que tanto os frutos tratados com etileno quanto os frutos do tratamento controle mantidos à temperatura de $20^{\circ} \mathrm{C}$ apresentaram alteração de coloração mais rápida de que aqueles tratados ou não com este fitohormônio e armazenados a $12,5^{\circ} \mathrm{C}$ (Figura 1). Constatou-se que a partir do nono dia de armazenamento a relação $a^{*} / b^{*}$, um indicativo da mudança de cor do verde para o vermelho, era significativamente inferior ( $\mathrm{pd} \leq 0,05)$ nos frutos armazenados a $12,5^{\circ} \mathrm{C}$ do que nos mantidos a $20^{\circ} \mathrm{C}$. Observou-se que os frutos armazenados a $20^{\circ} \mathrm{C}$, tratados ou 


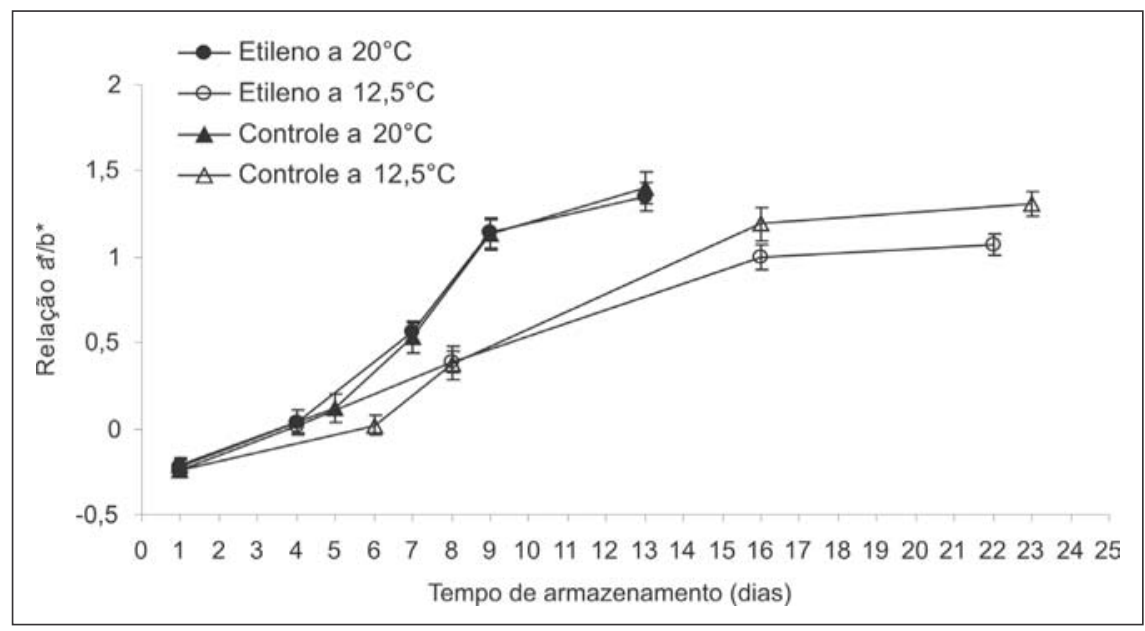

Figura 1. Relação $a * / b *$ de tomates 'Andréa' tratados com etileno e armazenados a $12,5^{\circ} \mathrm{C}$ e $20^{\circ} \mathrm{C}$. Barras verticais representam o desvio-padrão da média $\left(\mathrm{a}^{*} / \mathrm{b}^{*}\right.$ relation of tomato fruits, cv. Andrea, treated with ethilene and stored under $12.5^{\circ} \mathrm{C}$ and $20^{\circ} \mathrm{C}$. Vertical bars indicate the average standard deviation). Campinas, Unicamp, 2005.

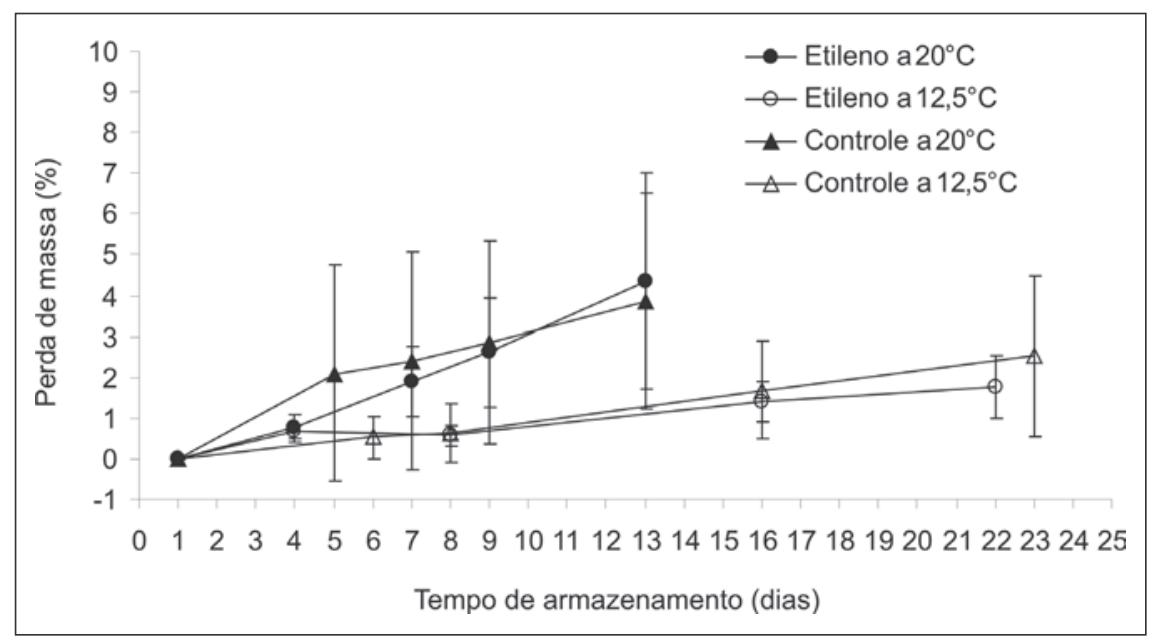

Figura 2. Perda de massa de tomates 'Andréa' tratados com etileno e armazenados a $12,5^{\circ} \mathrm{C}$ e $20^{\circ} \mathrm{C}$. Barras verticais representam o desvio-padrão da média (Mass loose from tomato fruits, cv. Andrea, treated with ethilene and stored under $12.5^{\circ} \mathrm{C}$ and $20^{\circ} \mathrm{C}$. Vertical bars indicate the average standard deviation). Campinas, Unicamp, 2005.

não com etileno, apresentavam-se no $13^{\circ}$ dia de armazenamento com sinais externos de podridão sendo, portanto, descartados. Tal alteração só ocorreu nos frutos mantidos a $12,5^{\circ} \mathrm{C}$ ao redor de 24 dias, comprovando-se o efeito benéfico da baixa temperatura na manutenção da qualidade dos tomates (Figura 1).

Os fatos observados em relação ao desenvolvimento da cor externa dos frutos tratados com etileno estão relacionados ao aumento da velocidade dos processos metabólicos que é estimulada pelo etileno exógeno (Davies \& Hobson, 1981). De maneira análoga, Moretti et al. (2002) verificaram que efeito da temperatura nos processos relacionadas à perda de água, via transpiração, e perda de matéria seca, via processo respiratório (Hobson \& Grierson, 1993; Nilsson, 2000). Verificou-se que a partir do sétimo dia de armazenamento os tomates tratados com etileno e mantidos a $20^{\circ} \mathrm{C}$ apresentaram um aumento significativo da perda de massa em relações aos frutos mantidos a $12,5^{\circ} \mathrm{C}$, com ou sem aplicação de etileno. Ao final do experimento a perda de massa de tomates mantidos a $20^{\circ} \mathrm{C}$ e tratados com etileno era $120 \%$ maior do que frutos não tratados com etileno e mantidos a $12,5^{\circ} \mathrm{C}$ (Figura 2).

De acordo com Andrade-Junior (1999), uma perda de massa equivalente de 3 a $6 \%$ já representa depreciação do produto. A maior perda de massa ocorreu para frutos mantidos a $20^{\circ} \mathrm{C}$ no $13^{\circ}$ dia de armazenamento (Figura 2). Frutos com aplicação de etileno apresentaram média de $4,35 \%$ e frutos do tratamento controle apresentaram média de 3,86\%. Tais resultados estão de acordo com Chomchalow (1991), que observou menor porcentagem de perda de massa para frutos de tomate 'Sunny' mantidos a $12,5^{\circ} \mathrm{C}$.

Verificou-se que a relação brix/acidez apresentou ligeira redução para os diferentes tratamentos até o $9^{\circ}$ dia de armazenamento para frutos a $20^{\circ} \mathrm{Ce} 16^{\circ}$ dia para frutos armazenados a $12,5^{\circ} \mathrm{C}$, mostrando um discreto crescimento nessa razão para frutos mantidos à temperatura inferior ao final do experimento (Figura 3). Tal comportamento é explicado tanto pelas alterações observadas para os teores de ácidos orgânicos quanto para os teores de sólidos solúveis totais que apresentaram tendências de redução até o $9^{\circ}$ e $16^{\circ}$ dia para frutos mantidos a 20 e $12,5^{\circ} \mathrm{C}$, respectivamente, com ligeira elevação destes teores até o final do período experimental (Figura 3).

A discreta redução verificada para a acidez titulável é explicada pela utilização de ácidos orgânicos, principalmente o ácido cítrico, predominante em tomates, como substrato respiratório na póscolheita de diversas frutas e hortaliças (Tucker, 1993). Castro (2000) e Castro (2003) também verificaram queda na acidez titulável durante o amadurecimento dos tomates para as cultivares Santa Clara e Carmem, respectivamente. 
De maneira similar, os teores de sólidos solúveis apresentaram discreta redução nos períodos mencionados anteriormente, que pode estar relacionada com o consumo de açúcares no processo respiratório, de acordo com Young et al. (1993) citado por Filgueiras (1996). O aumento no conteúdo desses sólidos próximo ao final do experimento está relacionado com a perda de massa, notadamente na forma de água, sofrida pelos frutos durante o armazenamento.

Pelos resultados obtidos no presente trabalho, verificou-se que a aplicação de etileno não demonstrou efeito significativo para a relação brix/acidez titulável em tomates 'Andréa', conforme já haviam relatado Kader et al. (1978) para cv. Cal Ace e ManzanoMendez et al. (1984) para cv. New Yorker. De maneira similar, Garcia et al. (1977) também verificaram que o etileno exógeno não exerceu efeito sobre os teores de sólidos solúveis totais.

Observou-se que houve tendência de aumento nos teores de ácido ascórbico para tomates tratados ou não com etileno e armazenados a temperatura de 20 e $12,5^{\circ} \mathrm{C}$ (Figura 4). Segundo Yahia et al. (2001), o conteúdo de ácido ascórbico é submetido a reações de oxidação e redução durante o amadurecimento do tomate. Os produtos de oxidação consistem de radicais livres do ácido, os quais podem ser revertidos novamente ao ácido ascórbico, indicando possibilidade de aumento desse composto ao longo do amadurecimento do fruto. Para os frutos mantidos a $12,5^{\circ} \mathrm{C}$ verificouse que no $6^{\circ}$ dia de armazenamento os teores de ácido ascórbico eram significativamente maiores em tomates do tratamento controle do que nos tomates tratados com etileno, um indicativo de que o hormônio vegetal possa ter contribuído para acelerar o processo de degradação do ácido ascórbico.

Diferença significativa similar foi observada no quarto dia de armazenamento para frutos tratados com etileno e mantidos a $20^{\circ} \mathrm{C}$, que possuíam teor menor de ácido ascórbico do que frutos do tratamento controle mantidos na mesma temperatura (Figura 4). Ao final do experimento verificou-se que tanto para os tomates armazenados a $20^{\circ} \mathrm{C}$ quanto para os mantidos a $12,5^{\circ} \mathrm{C}$

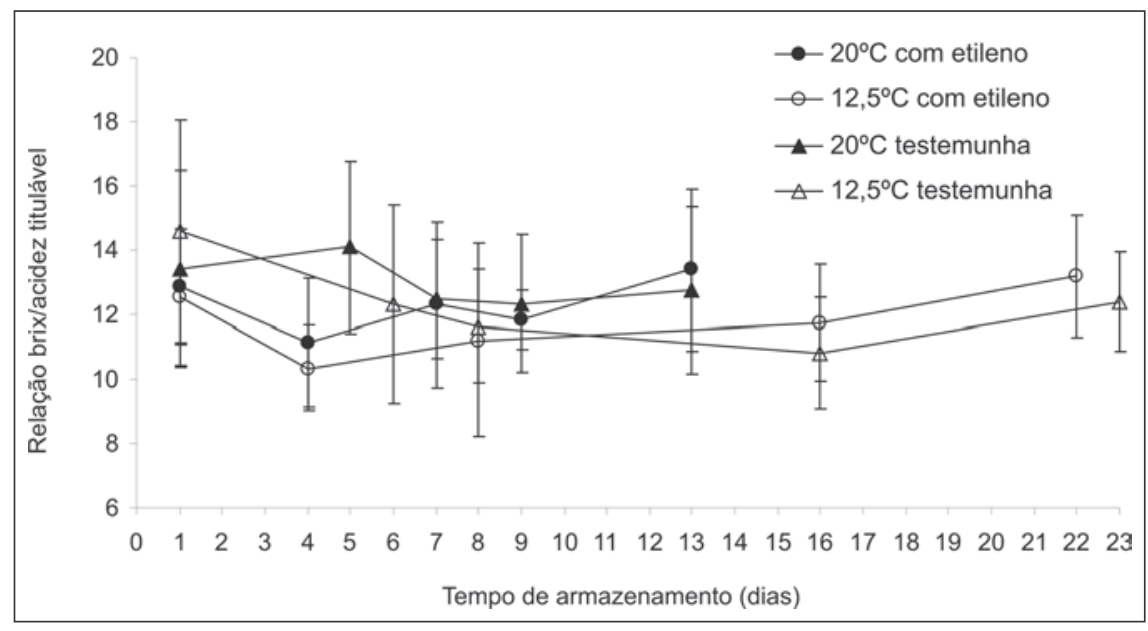

Figura 3. relação brix/acidez de tomates 'Andréa' tratados com etileno e armazenados a $12,5^{\circ} \mathrm{C}$ e $20^{\circ} \mathrm{C}$. Barras verticais representam o desvio-padrão da média (Brix/acidity relation of tomato fruits, cv. Andrea, treated with ethilene and stored under $12.5^{\circ} \mathrm{C}$ and $20^{\circ} \mathrm{C}$. Vertical bars indicate the average standard deviation). Campinas, Unicamp, 2005.

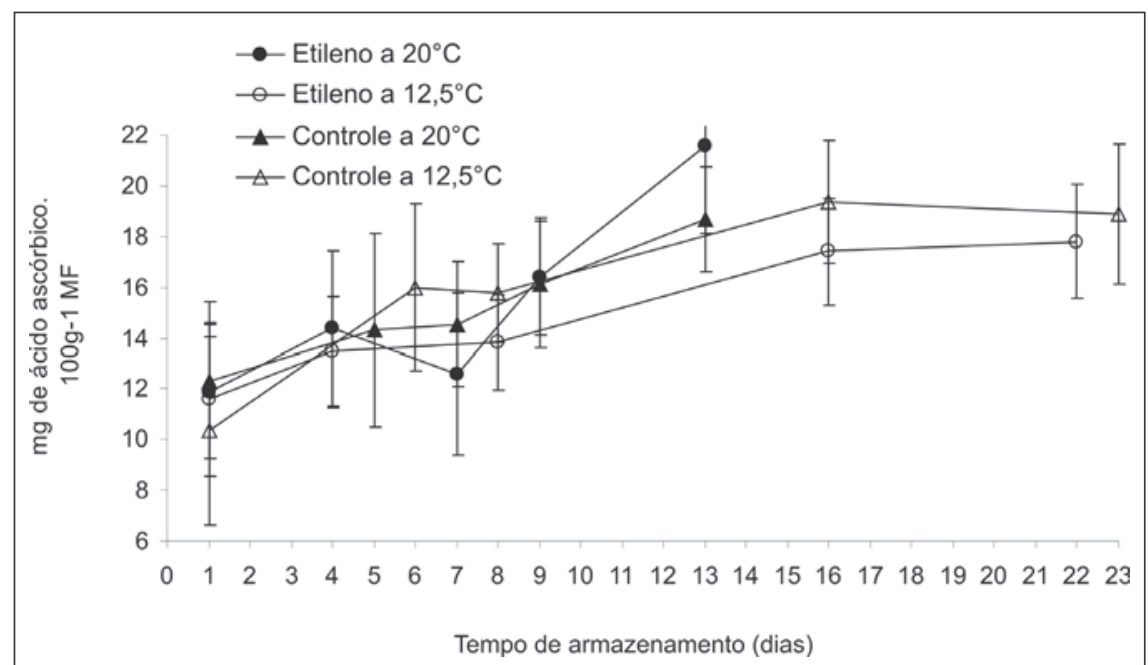

Figura 4. Teor de ácido ascórbico de tomates 'Andréa' tratados com etileno e armazenados a $12,5^{\circ} \mathrm{C}$ e $20^{\circ} \mathrm{C}$. Barras verticais representam o desvio-padrão da média (Ascorbic acid ?? of tomato fruits cv. Andrea, treated with ethilene and stored under $12.5^{\circ} \mathrm{C}$ and $20^{\circ} \mathrm{C}$. Vertical bars indicate the average standard deviation). Campinas, Unicamp, 2005. MF = matéria fresca.

não havia diferença significativa entre os frutos tratados ou não com etileno. Tais resultados estão de acordo com o verificado por Garcia et al. (1977) e Castro (2003) para a cv. Carmem. Por outro lado, Kader et al. (1978) encontraram diferença nos níveis de ácido ascórbico para tomates cv. Cal Ace com e sem aplicação de gás etileno, não afirmando, entretanto, qual a causa dessas alterações. Chomchalow (1991) encontrou valores mais elevados de ácido ascórbico em tomates cv. Sunny tratados com gás etileno.

$\mathrm{O}$ armazenamento a $20^{\circ} \mathrm{C}$ teve efeito tanto na velocidade quanto na extensão das modificações da cor dos tomates em comparação com o armazenamento realizado a $12,5^{\circ} \mathrm{C}$, não havendo contribuição do etileno exógeno na velocidade de ocorrência desse processo em ambas temperaturas estudadas. Tal informação pode ser útil a produtores que desejam acelerar o desenvolvimento da cor externa do fruto por razões mercadológicas. Todavia, pelos resultados obtidos nas demais variáveis químicas e físicas estudadas, concluise que a utilização de etileno exógeno em tomates 'Andréa' não é recomendável. 


\section{AGRADECIMENTOS}

À FAPESP e ao projeto PRODETAB/EMBRAPA pelo suporte financeiro e ao CNPq pela concessão da bolsa de mestrado.

\section{REFERÊNCIAS}

ALEXANDER L; GRIERSON D. 2002. Ethylene biosynthesis and action in tomato: a model for climacteric fruit ripening. Journal of Experimental Botany 53: 2039-2055.

ANDRADE JÚNIOR VC. 1999. Avaliação do potencial produtivo e da firmeza pós-colheita de frutos em híbridos de tomateiro. 52p. Dissertação (Mestrado em Genética e Melhoramento de Plantas) - Universidade Federal de Lavras, Lavras.

AOAC. 1984. Official Methods of Analysis. Association of Official Analytical Chemists, Washington, D. C. p. 1058-1059.

BENASSI MT; ANTUNES AJ. 1988. A comparison of metaphosphoric and oxalic acids as extractants solutions for the determination of vitamin $\mathrm{C}$ in selected vegetables. Arquivos de Biologia e Tecnologia 31: $507-513$.

CASTRO LR. 2000. Influência de aspectos de classificação, embalagem e refrigeração na conservação pós-colheita de tomate "Santa Clara" e "Carmem”. 159p. Dissertação (Mestrado em Tecnologia Pós-Colheita) - Universidade Estadual de Campinas, Campinas.

CASTRO VASPT. 2003. Controle do amadurecimento pós-colheita do tomate 'Carmem' tratado com ácido 2-cloroetil fosfônico. 76p. Dissertação (Mestrado em Tecnologia Pós-Colheita) - Universidade Estadual de Campinas, Campinas.

CARVALHO CRL; MANTOVANI DM; CARVALHO P R N; MORAES RM. 1990. Análises Químicas de Alimentos (Manual Técnico). Campinas: Biblioteca do ITAL, 145 p.

CHOMCHALOW S. 1991. Storage conditions and timing of ethylene treatment affect ripening uniformity and marketability of tomato fruits. 137p. Dissertação (Mestrado em Ciências) University of Florida, Florida.

DAVIES JN; HOBSON GE. 1981. The constituents of tomato fruit - The influence of environment, nutrition and genotype. CRC Critical Reviews in Food Science and Nutrition, Cleveland, v.15, n.3, p.205-208.

FILGUEIRAS HAC. 1996. Bioquímica do amadurecimento de tomates híbridos heterozigotos no loco 'alcobaça'. 118p. Tese (Doutorado em Ciências dos Alimentos) - Universidade Federal de Lavras, Lavras.
GALVIS-VANEGAS JA. 1987. Fisiologia póscolheita de tomate (Lycopersicon esculentum Mill) cultivar Ângela. 123p. Dissertação (Mestrado em Tecnologia Pós-Colheita) - Faculdade de Engenharia Agrícola, Universidade Estadual de Campinas, Campinas.

GARCIA JLM; BERNHART LW; SHIROSE I. 1977. Influência do ácido 2-cloroetilfosfônico (Ethrel) na maturação do tomate. Boletim do Instituto de Tecnologia de Alimentos, ITAL, 53: 141-157.

GULL DD. 1981. Ripening tomatoes with ethylene. Vegetable crops fact sheet. VC-29. Florida Cooperative Extension Service, Institute of Food and Agricultural Sciences, University of Florida. 4p.

HOBSON GE; GRIERSON D. 1993. Tomato. In: SEYMOUR GB; TAYLOR JE; TUCKER GA. (Eds.). Biochemistry of fruit ripening. London: Chapman \& Hall. p.405-442.

KADER AA; MORRIS LL; STEVENS MA; ALBRIGHT-HORTON M. 1978. Composition and flavor quality of fresh market tomatoes as influenced by some postharvest handling procedures. Journal of the American Society for Horticultural Science 103: 6-13.

KIEBER J. 2004. Etileno: o hormônio gasoso. In: TAIZ L; ZEIGER E. (Eds.). Fisiologia vegetal. $3^{\circ}$ ed. Porto Alegre: Artmed Ed. p.541-559.

MANZANO-MENDES J; HICKS JR; MASTER JF. 1984. Influence of storage temperature and ethylene on firmness, acids, and sugars of chilling-sensitive and chilling-tolerant tomato. Journal of the American Society of Horticultural Science 109: 273-277.

MAPA, Ministério da Agricultura, Pecuária e Abastecimento. Anexo XVII - Regulamento técnico de identificação e qualidade para a classificação do tomate, 2000. Disponível em: $<$ http://extranet.agricultura.gov.br/agrolegis/ Imagem? codArquivo=2472>. Acesso em 27 de janeiro de 2005

MAPA, Ministério da Agricultura, Pecuária e Abastecimento. Estatísticas. Disponível em: <www.agricultura.gov.br/pls/portal/docs/ PAGE/MAPA/ESTATISTICAS>. Acesso em 27 de janeiro de 2005.

McKEON TA; YANG SF. 1987. Biosynthesis and metabolism of ethylene. In: DAVIES PJ. (Ed.). Plant hormones and their role in plant growth and development. Dordrecht: Martinus Nijhoff Publishers. p.94-112.

MORETTI CL; SARGENT SA; HUBER DJ; CALBO AG; PUSCHMANN R. 1998. Chemical composition and physical properties of pericarp, locule and placental tissues of tomatoes with internal bruising. Journal of The American Society for Horticultural Science 123: 656-660, 1998.
MORETTI CL; ARAUJO AL; MAROUELLI WA; SILVA WLC. 2002. 1methylcyclopropene delays tomato fruit ripening. Horticultura Brasileira 20: 659-663.

NILSSON T. 2000. Postharvest handling and storage of vegetables. In: SHEWFELT RL; BRUCKNER B. (Eds.) Fruit \& Vegetables Quality. An integrated view. Lancaster: Technomic Publishing Company Inc. Cap. 6, p. $96-121$.

REID MS. 1994. Biology of ethylene production and action. Perishables Handling Newsletter. Issue n. 80 .

REID MS. 2002. Ethylene in postharvest technology. In: KADER AA. (Ed). Postharvest Technology of Horticultural Crops. Third Edition, University of California. Division of Agriculture and Natural Resources Publication 3311. p. 149-162.

SALTVEIT ME Jr. 1999. Effect of ethylene on quality of fresh fruits and vegetables. Postharvest Biology and Technology 15: 279-292.

SARGENT SA, MORETTI CL. 2004. Tomato In: The Commercial Storage of Fruits, Vegetables, and Florist and Nursery Crops. Beltsville, Maryland, EUA: USDA. 1: 1-7. Disponível em http://www.ba.ars.usda.gov/hb66/index.html (Acesso em 19 de Dezembro de 2005).

SHAMAILA M; POWRIE WD; SKURA BJ. 1992. Sensory evaluation of strawberry fruit stored under modified atmosphere packaging (MAP) by quantitative descriptive analysis. Journal of Food Science 57: 1168-1172.

STEVENS MA. 1985. Tomato flavor: effects of genotype, cultural practices, and maturity at picking. In: PATEE HE. (Ed.). Evaluation of quality of fruits and vegetables. Westport: AVI Publ. 410p.

SUSLOW TV; CANTWELL M. 2003 Produce Facts Tomato: Recommendations for Maintaining Postharvest Quality. In: UC Davis, Postharvest technology: research and information center. Management of Fruit Ripening. Postharvest Horticulture 9: 91-93.

TUCKER GA. 1993. Introduction. In: SEYMOUR GB; TAYLOR JE; TUCKER GA (Eds.). Biochemistry of fruit ripening. London: Chapman \& Hall. p.01-51.

YAHIA EM; CONTERAS-PADILLA M; GONZALEZ-AGUIAR G. 2001. Ascorbic acid content in relation to ascorbic acid oxidase activity and polyamine content in tomato and bell pepper fruits during development, maturation and senescence. Lebensm.-Wiss und Technol. 34: 452-457 